\title{
Investigations médicales préalables indépendantes
}

\author{
Barbara Züsta ${ }^{a}$ Lotte Arnold-Graf \\ a lic. iur., directrice adjointe et responsable du domaine professionnel de l'Organisation suisse des patients (OSP) \\ ${ }^{b}$ Directrice de I'Organisation suisse des patients (OSP)
}

\begin{abstract}
Depuis 34 ans, les patients peuvent adresser leurs questions à l'Organisation suisse des patients (OSP). S'ils soupçonnent une faute de traitement, les déclarations des médecins chargés du suivi peuvent les induire en erreur. Dans ce contexte, les experts médicaux procèdent à de premières investigations pour analyser les faits et pondérer les reproches. Il est cependant rare qu'un soupçon aboutisse à l'ouverture d'une procédure.
\end{abstract}

\section{Historique de l'OSP}

Un mur mobile a été mis en place pour la première fois à Zurich en 1978 sous l'impulsion du Gottlieb Duttweiler Institut dans le cadre d'une action citoyenne [1] dans le but de recueillir les messages de patients insatisfaits. Suite à un nombre inattendu de plaintes déposées par des patients lésés, qui à l'époque intéressaient aussi les médias, le premier bureau des patients voyait le jour en 1979 en Suisse alémanique, avant de devenir un pionnier pour toutes les organisations de patients, même en Allemagne.

Fondée en 1981, l'«Organisation suisse des patients (OSP)» a été la première organisation de patients politiquement indépendante à agir au niveau national. Si au début, elle traitait près de 400 appels téléphoniques par an, ce sont aujourd'hui pas moins de 4000 personnes qui contactent nos antennes de consultation à Zurich (secrétariat), Bellinzone, Berne, Genève, Lausanne, Olten ou St-Gall.

\section{Formation et information des patients}

Nous conseillons et soutenons les patientes et les patients en leur proposant des séances d'information et de formation [2] dans le but de leur transmettre des informations compréhensibles et des explications médicales accessibles afin qu'ils puissent décider par euxmêmes selon leurs propres préférences.

Pour épauler les patients et leurs proches, nous avons développé un guide de conseil pratique «Patientenkompass», disponible en librairie et par voie électro- nique à partir de juin 2015 [3]. En plus d'offrir une multitude d'informations utiles, cette brochure guide le patient dans les cinq étapes du traitement, lui suggère d'y participer de manière proactive et de discuter d'égal à égal avec le corps médical.

L'enthousiasme des médecins n'est pas toujours au rendez-vous lorsqu'ils reçoivent des patients qui ont recueilli un grand nombre d'informations sur internet avant de se présenter à la consultation. Nous encourageons les médecins à considérer ce comportement comme une prédisposition positive du patient à vouloir se prendre en main, même si au cas par cas, les informations glanées sur la toile risquent de plus le perturber que de l'informer. Un patient qui joue un rôle actif et sensé dans une prise de décision est plus à même de participer durablement au processus thérapeutique car il acquiert davantage de compréhension pour le traitement recommandé. De la même manière, il développe des attentes plus réalistes quant aux objectifs du traitement.

L'Organisation suisse des patients (OSP) se finance à $50 \%$ par les recherches qu'elle effectue au préalable et les conseils aux patients. La deuxième moitié du financement de son activité provient des cotisations de l'Association des donateurs, de dons privés et de subventions cantonales.

Si vous souhaitez soutenir le travail de I'OSP en faveur des droits et de la protection des patients, devenez membre de I'Association des donateurs. Madame Lotte Arnold, directrice de I'OSP, atteignable à lotte.arnold[at]spo.ch ou au tél. 044 2525422 , se réjouit de votre prise de contact. 


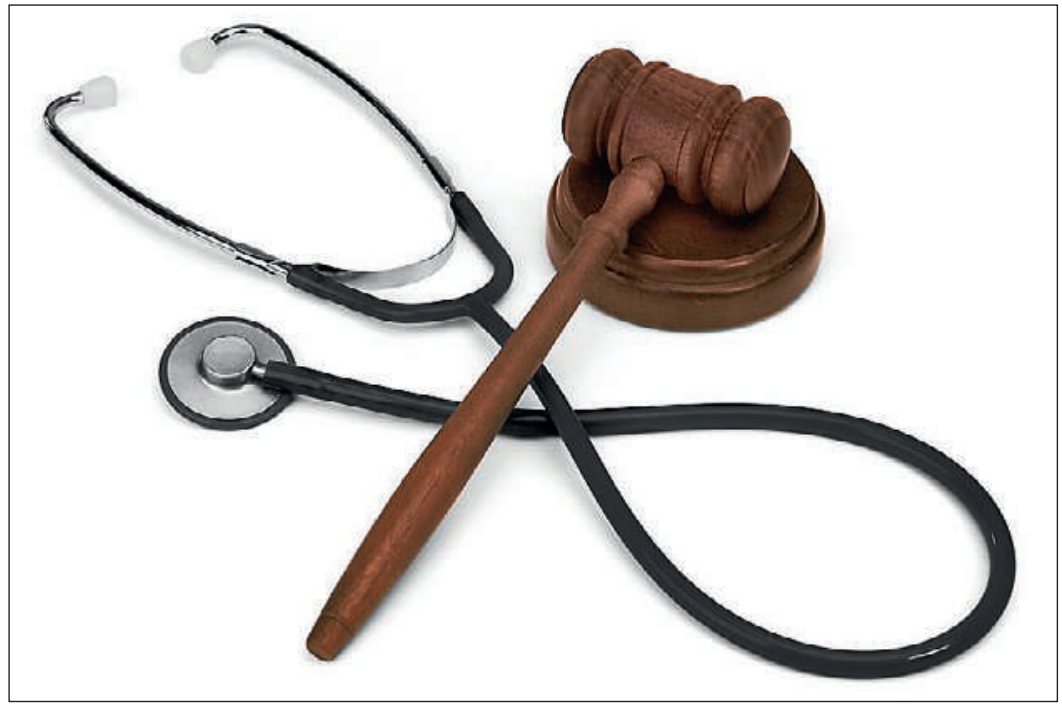

Seulement très peu de dossiers sont envoyés à un juge civil, encore moins à un juge pénal.

\section{Conseil lors d'une faute de traitement présumée}

Nombreux sont ceux qui soupçonnent une faute de traitement si leurs attentes ne se concrétisent pas après une opération. Le manque de diligence du médecin (faute de traitement) ne constitue cependant pas l'unique cause d'un résultat indésirable, il est également possible qu'un risque non évitable se produise (complication).

Nous recommandons aux patients qui soupçonnent une faute de traitement ou de diagnostic à prendre contact avec le médecin qui les a traités en vue d'obtenir de plus amples informations concernant le déroulement du traitement qui a conduit à un résultat indésirable. Si cet entretien ne devait pas apporter les clarifications nécessaires, il est alors indiqué de demander conseil à un expert médical neutre.

\section{Le rôle des médecins chargés du suivi}

Notre expérience nous a montré que des remarques ou déclarations comme "Je n'aurais certainement pas opéré comme ça» ou "Vous seriez venu me voir..." prononcées de manière irréfléchie par les médecins chargés du suivi, peuvent parfois déstabiliser les patients. Dans de telles situations, il faut faire très attention car ces derniers peuvent les interpréter, souvent par erreur, comme un indice les confortant dans leur sentiment d'être victimes d'une faute de traitement et les incite à la confrontation avec le médecin. Mais de manière générale, le "combat» ne vaut la peine que dans les cas où les patients présument un manque de diligence à l'origine des atteintes à leur santé et des pertes subies.

\section{Investigations médicales préalables de l'OSP}

Si un patient s'adresse à nous - une organisation neutre et indépendante dotée des experts requis -, nous procédons à des investigations préalables pour examiner si les soupçons de faute sont avérés. Pour cela, nous demandons tout d'abord le dossier du patient complet au médecin traitant. Il peut arriver que les médecins qui nous transmettent le dossier souhaitent être informés des recherches en cours de l'OSP et des prochaines étapes. Dans ce cas, nous leur rappelons que nous défendons les intérêts du patient à sa demande explicite et qu'à ce titre, nous sommes soumis au devoir de fidélité et de confidentialité pour ce qui nous a été confié. Sans accord explicite du patient, nous ne pouvons donc donner aucun renseignement.

\section{Suite de la procédure}

Il est important de savoir qu'en premier lieu, nous déterminons les faits en constituant un dossier et en posant des questions à nos médecins-conseils. Il ne s'agit pas pour nous de prononcer des reproches ou de faire valoir des demandes de réparation.

D'éventuelles mesures supplémentaires ne sont décidées qu'à l'issue d'un entretien final qui se déroule avec l'avocat-conseil et le patient, trois à six mois environ après le début de nos recherches. La plupart des cas

\section{Nous recommandons aux patients qui} soupçonnent une faute de traitement ou de diagnostic à prendre contact avec le médecin.

(75\% environ) sont classés sans suite. Si les parties concernées, c'est-à-dire l'avocat ou l'assurance responsabilité civile, ne se manifestent pas dans les six à neuf mois chez le médecin, celui-ci peut en conclure que le cas ne sera pas poursuivi. S'il a des questions pendant cette période, nous préférons qu'il ne s'adresse pas au patient mais au conseiller de l'OsP, qui est le premier interlocuteur pendant les investigations. En règle générale, cette manière de procéder est ressentie comme un soulagement par le patient

Nous identifions environ 150 cas éventuels de responsabilité civile par an, que nous transmettons au terme de nos recherches à nos avocats-conseils spécialisés [4].

\section{Eviter les procédures pénales}

Il est primordial que d'éventuels cas de responsabilité civile soient traités par des avocats expérimentés car les avocats non spécialisés risquent d'impliquer méde- 
cins et patients dans des procédures longues et astreignantes ne menant à aucun résultat. Comme nous sommes en contact avec des avocats-conseils pendant nos recherches préalables et que nous ne collaborons qu'avec des avocats expérimentés, nous sommes en mesure de "filtrer» l'essentiel des nombreux reproches exprimés par les patients. Nous prenons le temps de commenter les faits avec eux et, grâce à la confiance qu'ils nous accordent, nous pouvons leur expliquer de manière crédible pourquoi certains risques peuvent subsister malgré toute la diligence du médecin. De ce fait, nous évitons de nombreux désagréments aux médecins concernés. Par ailleurs, médecins et patients font l'économie de dépenses et de frais de procédures inutiles.

\section{Rôle central de la communication}

Une communication lacunaire est souvent un élément déclenchant pour que les personnes concernées viennent chercher des explications sur ce qui leur est

\section{Nous identifions environ 150 cas éventuels de responsabilité civile par an.}

arrivé et de l'aide auprès d'un organisme indépendant. Une intervention de l'OSP en tant que porte-voix des patients, et une invitation à une séance de médiation peuvent parfois faire des merveilles chez tous les protagonistes.

Si le dialogue entre le patient et le médecin revêt de l'importance, il n'a jamais été aussi difficile qu'aujourd'hui. En effet, la spécialisation et la sous-spécialisation de la médecine engendre une énorme répartition des tâches au détriment de la relation humaine. D'une part, les patients et les médecins ont de moins en moins de temps pour discuter et, de l'autre, les exigences en matière de communication augmentent des deux côtés, aussi bien pour le médecin que pour le patient. Si le dialogue n'est plus possible, une aide extérieure comme celle des organisations de patients peut s'avérer extrêmement fructueuse.

Correspondance:

Barbara Züst

Organisation suisse

des patients (OSP)

Häringstrasse 20

$\mathrm{CH}-8001$ Zurich

Tél. 0442525422

Fax 0442525443

barbara.zuest[at]spo.ch

\section{L'OSP ne recommande (pratiquement) jamais une procédure pénale}

Nous avons conscience que la critique de leur travail met les médecins à rude épreuve. Mais il est important de savoir que $98 \%$ des cas de responsabilité civile se terminent par une procédure extrajudiciaire. Seule-

\section{Le Bureau d'expertises extrajudiciaires} de la FMH en bref

Le Bureau d'expertises extrajudiciaires de la FMH permet aux patients qui soupçonnent une faute de diagnostic et/ou de traitement de faire organiser - sous certaines conditions - une expertise extrajudiciaire par un expert neutre et compétent. La procédure est transparente et régie par un règlement. Dans ce cadre, les patients peuvent s'adresser au Bureau d'expertises pour s'informer sur les démarches à effectuer lorsqu'ils pensent avoir été victimes d'une faute médicale. Ils peuvent également obtenir des modèles de documents (notamment de demande), qui facilitent leur participation à la procédure. Le Bureau d'expertises reçoit aussi des cas qui ont été préparés par l'Organisation suisse des patients (OSP) et apprécie grandement le travail effectué en amont par cette dernière. En effet, en plus du fait que le travail de l'OSP permet de soulager le patient de plusieurs démarches à effectuer, cette organisation effectue des recherches préalables qui permettent de filtrer les dossiers de manière efficace. Ainsi, les cas qui parviennent au Bureau d'expertises sont bien documentés. Par ailleurs, la $\mathrm{FMH}$ et I'OSP ont collaboré à la rédaction d'une brochure intitulée Communication entre médecin et patient - Recommandation en cas d'incident médical, parue en juin 2014.

ment très peu de dossiers sont envoyés à un juge civil, encore moins à un juge pénal. Les avocats de l'OSP ne recommandent que dans de très rares cas de lancer une procédure pénale, par exemple si le dossier du patient a été manipulé. Les procédures pénales qui ont pour objet un reproche de culpabilité personnel créent plus de dégâts qu'elles n'en enlèvent, car elles durcissent inutilement les fronts et laissent peser une énorme pression sur les médecins.

Le risque est inhérent à tout traitement médical et les médecins font des erreurs comme tout être humain. Il est important de recourir contre les fautes médicales mais il ne faut pas que cela incite les médecins à refuser des patients difficiles ou à exercer une médecine défensive. Nous démontrons qu'une bonne gestion des conflits entre les patients et les personnes impliquées, et un comportement ouvert face à la faute médicale, peuvent aider à aller au-delà des reproches.
Références

1 Schweizer Frauenblatt, 6/1983, page 7

2 Exemple de la manifestation de l'université des séniors organisée à Zurich-Irchel au printemps 2015.

3 ISBN 978-3-905795-40-0, brochure de 114 pages, CHF 29.90, e-book, CHF 19.90 (en allemand uniquement).

4 En collaboration avec nos avocats-conseils, nous avons élaboré un flyer à l'intention des médecins et des patients intitulé L'expertise médicale, qui peut être commandé auprès de l'OSP. 